\title{
Holzkohlenreste der Seesedimente von Kathmandu
}

\section{H. Boesch: Lage und geologisch-morphologische Beschreibung}

In den Jahren 1968 bis 1973 führte der Autor in Nepal und besonders im Tal von Kathmandu morphologische Untersuchungen durch. In einem auch die bibliographischen Hinweise auf vorläufige Teilberichte enthaltenden Schlußbericht, erschienen in Geographica Helvetica 1974 , p.15-26, wurde eine auf dem Feldbefund beruhende Arbeitshypothese zur Morphogenese des Tales von Kathmandu vorgelegt.

Die genannten Untersuchungen erfolgten vor allem im südlichen Teil des Tales in detaillierter Weise, während Gebiete nördlich der Hauptstadt nur kursorisch begangen wurden. Dies geschah, weil der Schlüssel zum Verständnis im Süden und nicht im Norden zu finden ist. Im Norden erheben sich über dem 1200 bis $1400 \mathrm{~m}$ ü. M. gelegenen Tal von Kathmandu die aus präkambrischen, meist sauren magmatischen $\mathrm{Ge}$ steinen aufgebauten Gipfel der Sheopuri Lekh auf über $2500 \mathrm{~m}$ ü. M. Das Verwitterungsmaterial wurde und wird in der Form von Sanden vom Fuße der Sheopuri Lekh bis weit ins Talbecken hinaus abgelagert. Die stratigraphischen Verhältnisse mit Deltaschüttungen, synsedimentären Rutschungen usw. lassen keinen Zweifel daran aufkommen, daß diese Aufschüttungen einmal in stehenden Wasserkörpern erfolgten. Der rasche Wechsel der stratigraphischen Verhältnisse läßt vermuten, daß es sich kaum um einen zusammenhängenden tiefen See, sondern wohl eher um eine Serie von flachen Wannen, Sümpfen usw. gehandelt haben muß. Die Sande sind streckenweise sauber gewaschen und fast reine Quarzkörnchen. Nur wenig entfernt nimmt der Anteil der Feldspäte stark zu, dann schalten sich tonige, lehmige Zwischenlagen ein und oft sind diese auch durch organische Beimengungen verfärbt. An manchen Stellen ist dieses organische Material lokal so angereichert, daß das Gestein in getrocknetem Zustand als Brennmaterial Verwendung findet.

Am 21. März 1973 führte ich nördlich von Kathmandu eine vergleichenden Untersuchungen dienende Exkursion durch. Ich verließ Kathmandu auf der gegen Nordwesten führenden Trisulistraße und zweigte bei Balaju von dieser in nördlicher Richtung ab. Eine schlechte aber fahrbare Straße führt vorerst über den Bishnumati Khola und erreicht kurz darauf ziemlich steil eine ca. $35 \mathrm{~m}$ höher gelegene Terrassenfläche.
Diese Terrassenfläche steigt nordwärts gleichmäßig sanft gegen den Gebirgsfuß an, etwa $30 \mathrm{~m}$ auf rund $4 \mathrm{~km}$. Auf ihr liegen die Dörfer Dandagoon und Phutung. An zwei Stellen wird bei Phutung die Terrassenfläche von Westen und Osten durch rückgreifende Erosion aufgezehrt.

$\mathrm{Ob}$ es sich bei der Terrassenfläche um eine Akkumulations- oder Erosionsoberfläche handelt, läßt sich auf dieser Strecke nicht mit Sicherheit entscheiden. Die Terrassenkanten sind scharf ausgebildet, doch verhindert die starke Bewachsung der Terrassenhänge eine eindeutige Aussage. Ich würde eher der Auffassung zuneigen, daß es sich um die Akkumulationsfläche handelt. Nicht zuletzt führte mich zu dieser Annahme das allmähliche Übergehen der Terrassenfläche nördlich von Phutung in die sehr steilen Schuttfächer bei Sangla.

Kathmandu bezieht einen Teil seines Wassers aus dem eben genannten Gebiet und eine Wasserleitung, die sich in sehr schlechtem Zustand befindet, verläuft parallel mit der Straße über Phutung südlich zur Hauptstadt.

Über die zeitliche Einordnung der erwähnten Ablagerungen und Formen wurde im eingangs erwähnten Bericht arbeitshypothetisch folgendes geschrieben: Die «Seeablagerungen» dürften in die letzte Eiszeit und in ihren oberen Teilen in das Würm-Spätglazial zu stellen sein. Ihre Zerschneidung erfolgte in verschiedenen Phasen, wobei aufgrund neuester ${ }^{14} \mathrm{C}$-Bestimmungen rund $5000 \mathrm{BP}$ - vermutlich durch tektonische Bewegungen im südlichen Teil des Kathmandu-Beckens verursacht - eine Aufstauung des Baghmati und eine Ablagerung der «Kalimati» genannten Tone in die geschaffenen Talsysteme hinein erfolgte. Eine leider nach Lage und Einordnung im stratigraphischen Profil nicht genauer beschriebene ${ }^{14} \mathrm{C}$-Bestimmung aus den Seeablagerungen selbst ergab $29115 \pm 32225$. Genauere Angaben dazu finden sich in Geographica Helvetica 1974.

Am 21. März 1973 wurde ich in Phutung auf einen Haufen zum Trocknen ausgelegter «Lignite» aufmerk-

Prof. Dr. H. Boesch, Geographisches Institut Universität, Blümlisalpstraße 10, 8006 Zürich.

C. A. Burga, dipl. nat., Nationalfonds-Assistent, Botanisches Institut Universität Basel, Schönbeinstraße 6, 4056 Basel. W. A. Keller, dipl. nat., Geographisches Institut Universität, Blümlisalpstraße 10, 8006 Zürich 
sam. Ich stieg dann in das kleine Tälchen hinunter, welches in westlicher Richtung dem Bishnumati Khola tributär ist. In einem Bambushain, wo Vegetation die geologischen Zusammenhänge verdeckte, waren eine größere Zahl von Männern, Frauen und Kindern damit beschäftigt, kohliges Material in einer Grube abzubauen. Dieses war von Wasser völlig durchtränkt; die Arbeit des Abbauens und Wegschaffens war schwer. Die Grube lag etwa $15 \mathrm{~m}$ tiefer als die Straße auf etwa $1355 \mathrm{~m}$ ü. M. Die Mächtigkeit der Lignitlinse schätzte ich auf rund $150 \mathrm{~cm}$, darüber folgten Sande und Gerölle, die schlecht gerundet waren und von denen die größten einen Durchmesser von $40 \mathrm{~cm}$ und mehr besassen. Ich nahm eine Probe mit in der Absicht, sie später einmal genauer zu untersuchen. Aus verschiedenen Gründen verzögerte sich diese Untersuchung. Es war aber möglich, in der Zwischenzeit Veröffentlichungen und Dokumente auszuwerten, die mir 1973 noch nicht zur Verfügung standen.

Fig. 1 wurde nach eigenen Beobachtungen und nach S. P. NAUTIYAL und P. N. SHARMA: Geological Map of Kathmandu Valley, 1:63360 sowie W.H.O. Project Nepal 0025, Drawing 4.4.5, 1973 gezeichnet. Legende: (1) Bhimphedi Serie, präkambrisch; Granite, Gneise, Migmatite, Schiefer, Quartzite und Kalke (2) ChitlangFormation, kambrisch-ordovizisch; hier hauptsächlich Kalke (3) Schuttfächer (4) meist sandige Seeablagerungen, pleistozän (5) Kalimati, subrezente Tone mit viel organischem Material (6) die im Text erwähnte Terrassenfläche, möglicherweise spätglaziale Akkumulationsfläche (7) Fundstelle der Kohlenreste (8) Lage der nachfolgend beschriebenen Bohrung No. BH 7 (9) Straßen und Fahrwege, Auswahl.

Über die geologischen Verhältnisse orientieren die folgenden neuesten Quellen: C. K. SHARMA: Geology of Nepal, Kathmandu, 1973 (die oben erwähnte geologische Karte ist jedoch eine nicht im Handel erschienene Lichtpause) sowie in erster Linie der von der World Health Organisation 1973 veröffentlichte Bericht zum Project Nepal 0025, Appendix 4.4 Geology of the Kathmandu Valley und Appendix 4.5 Groundwater Investigations. Diese Untersuchungen wurden von Binnie and Partners, London, durchgeführt. Abbildung 4.4.6 enthält ein aufgrund zahlreicher Bohrungen und Feldbeobachtungen gezeichnetes Profil, welches geologisch gegenüber den früher gemachten Angaben in unserem Abschnitt kaum Neues bringt. Dagegen wird hier die Terrassenfläche als eine

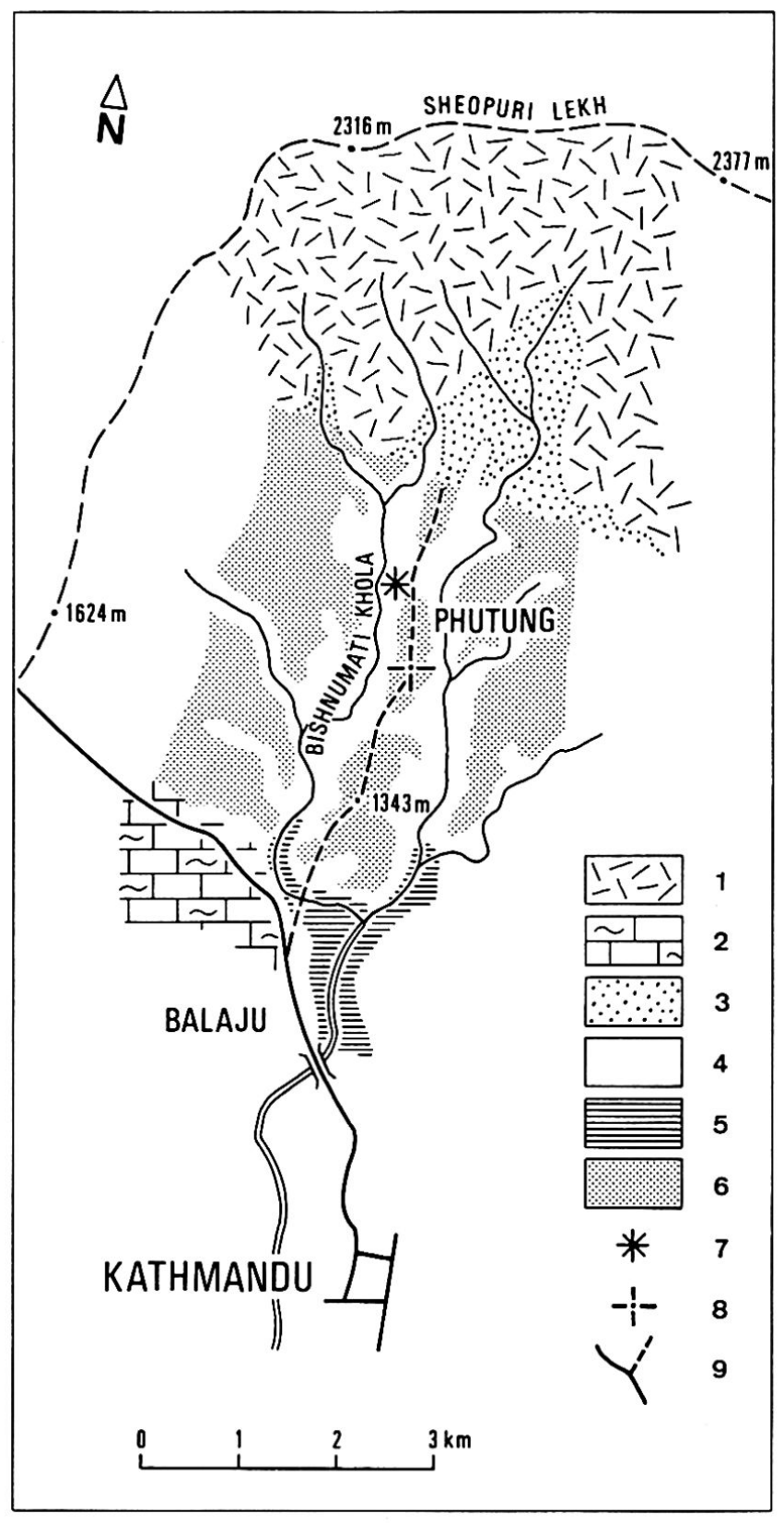

Fig. 1 Lageskizze (siehe Text)

Erosionsoberfläche gezeichnet, welche die Schichten schneidet.

Eingehende Angaben liefert der WHO-Bericht über eine Bohrung, welche im Jahre 1965 vom Geological Survey of India im Rahmen des Indian Aid Mission 
Programme etwa $800 \mathrm{~m}$ südlich unserer Fundstelle in Phutung ausgeführt wurde. Leider wird die Höhe über Meer der Bohrstelle nicht angegeben; sie dürfte nach meinen Aneroidbestimmungen und aufgrund der Karte 1:63360 $1365 \mathrm{~m}$ ü. M. betragen. Diese Bohrung besitzt die laufende Nummer «BH 7». Gebohrt wurde bis auf eine Tiefe von 201,61 m. Über die uns interessierenden Abschnitte gibt das Protokoll die folgende Auskunft:

00,00- 6,70 m: Oberflächenboden und Sand mit eingeschaltetem Tonlager

6,70-12,80 m: Grobsande von Orthogneisen, entsprechende Gerölle und in den tieferen Lagen Tone

12,80-58,21 m: Tone, tiefschwarz, kohlehaltig, glimmerreich, plastisch, vermischt mit Gneisgeröllen und Geröllagen

usw.

Die Übereinstimmung mit unserem Feldbefund ist groß.

Über die heutigen Vegetationsverhältnisse orientieren uns - freilich summarisch - in Text und Karte die vom CNRS herausgegebenen «Cahiers népalais-documents no. 4: carte écologique du Népal, région kathmandu-everest, 1/250 000». Der Autor (J.-F. DOBREMEZ) unterscheidet (1) im Becken von Kathmandu «étage subtropical inférieur, forêt mésohygrophile (Schima wallichii, Castanopsis indica, Engelhardtia spicata, Myrsine capitellata, Saurauja nepalensis)» (2) auf trokkeneren Gehängen der umgebenden Berge «forêt xérophile de l'étage subtropical inférieur (Pinus roxburghii, Phyllanthus emblica, Semecarpus anacardium)» (3) die im Norden liegenden Gebirge gehören in den unteren und mittleren Lagen zur collinen Stufe mit Quercus lanata und in den oberen Lagen zur Gebirgsstufe, hier mit Wäldern charakterisiert durch Quercus semecarpifolia. Mit Bezug auf das Becken und die Randgebiete schreibt der Autor: «... Tous ces groupements... ont été entièrement défrichés par l'homme. La zone subtropicale à très forte densité humaine est la zone du culture du riz. Les forêts subtropicales sont extrêmement rare.»

Herr C. A. BURGA übernahm die Bestimmung der Pollen der aufgesammelten Probe, Herr W. A. KELLER die ${ }^{14} \mathrm{C}$-Bestimmung. Ihre Untersuchungen erfolgten unabhängig voneinander und ohne jede weitere Auskunft über Fundort usw. Ihre Untersuchungsberichte werden so wiedergegeben, wie sie abgefaßt wurden. Sie fügen sich ausgezeichnet in das arbeitshypothetische Bild und stützen die aufgestellte These weitgehend. Sie zeigen aber auch, da $\beta$ weitere Untersuchungen - vor allem; wenn die Kalimati-Schichten einbezogen würden - Resultate versprechen, die auch mit Bezug auf die frühe Besiedelungsgeschichte und die entsprechenden Kulturpflanzen höchst interessant wären.

\section{A. Burga: Pollenanalytische Untersuchungen (Durchgeführt am Botanischen Institut der Uni- versität Basel)}

\section{Methodisches}

Etwa $1 \mathrm{~cm}^{3}$ der Holzkohlenprobe wurde zu Pulver zermörsert und nach der üblichen Aufbereitungsmethode $(10 \% \mathrm{KOH}$, Azetolyse, Glycerin-Präparate), vgl. BEUG, H.-J. (1961), behandelt.

Die mikroskopische Bestimmung der Pollen und Sporen erfolgte bei einer Vergrößerung von $200 \mathrm{mal}$, genauere Bestimmungen bei stärkeren Vergrößerungen mit Ölimmersion. Insgesamt wurden drei Präparate (Gesamtflächenanalysen) ausgezählt.

\section{Zur Bestimmung der Pollen und Sporen}

$\mathrm{Da}$ einschlägige Pollen- und Sporenbestimmungsliteratur der Nepalesischen Flora fehlt, konnten viele Pollen und Sporen nicht mit Sicherheit bestimmt werden, so daß eine Unterscheidung von Baum-, Strauchund Krautpollen nur bedingt richtig sein kann.

Als sicher identifizierte Pollen und Sporen können gelten:

Pinus spec.

Gramineae

Chenopodiaceae

Caryophyllaceae

Rubiaceae

Artemisia

Thalictrum

Compositae liguliflorae und C. tubuliflorae

Pteridophyta: Dryopteris-Typ 
Bei den Caryophyllaceen konnten zwei deutlich verschiedene Typen festgestellt werden, nämlich ein groBer Pollentypus (30-45 $\mu$, wenigporig) und ein kleinerer Typ (18.5-21 $\mu$, vielporig: 25-35 Poren). Die übrigen Pollen wurden aufgrund ihrer Ähnlichkeit mit mitteleuropäischen Pollenkörnern wie folgt bezeichnet (im Pollenspektrum mit Anführungszeichen versehen):

«Abies»:

Grobnetzige Luftsäcke, Pollenmittelteil mit Kamm, Gesamtlänge ca. $180 \mu$.

«Picea»: $\quad$ Luftsäcke feinnetziger und etwas diffuser in der Skulptur, Pollenmittelteil ohne deutlichen Kamm, Gesamtlänge ca. $170 \mu$.

«Larix»: $\quad \pm$ psilat, ohne Öffnung, ca. $180 \mu$ groß.

Pinus «Cembra»: größer als Pinus spec., Unterscheidungsmerkmale zu Pinus silvestris / montana / Mugo vgl. BEUG, H.-J. (1961).

"Quercus»: $\quad$ prolat, 3-colpat, verrucat, ca. 20 bis $35 \mu$ groß.

«Salix»: $\quad$ prolat, 3-colpat, perreticulat, ca. 20 bis $30 \mu$ groß.

«Corylus»: 3-porat, Poren ohne Vestibulum, aber dünnwandiger als Corylus, 25 bis $30 \mu$ groß.

«Cyperaceae»: feinverrucate Skulptur, ohne sichtbare Öffnungen (evtl. Dünnstellen?), unregelmäßiger Umriß des Pollenkorns, ca. $40-50 \mu$ groß.

Weitaus der größte Teil des organischen Materials besteht aus Holztrümmern, denen relativ viel mineralischer Detritus, besonders Silikate, beigemengt ist. Vier Nadelholz-Spaltöffnungen, Markstrahl-Querschnitte u. a. lassen vorwiegend auf Nadelholztrümmer schließen, was die Analyse der Pollen bestätigt.

\section{Zur Deutung des Pollenspektrums (vgl. Fig. 2)}

Am auffälligsten ist die Diskrepanz zwischen dem außerordentlichen Reichtum an Holztrümmern und andern organischen Resten und der Pollenarmut. Be- trägt doch die Pollenfrequenz nur 25 Pollen und Sporen pro $\mathrm{cm}^{2}$. Dem reichlichen Vorkommen von Holzresten sollte am ehesten eine hohe Pollenfrequenz entsprechen, und zwar besonders häufiges Vorkommen von Baumpollen.

Aber das Pollenspektrum deutet mit 52.6\% Nichtbaumpollen (12.2\% Baumpollen und $0.6 \%$ Strauchpollen) auf baumarme, wenn nicht sogar baumlose Rasenvegetation.

Die am stärksten vertretenen Krautpollen sind die Caryophyllaceen (18.1\%), die neben feuchteren Standorten vorwiegend Trocken- und Magerrasen, Steinschutt, Heiden und Neuland besiedeln. Dann folgen die «Cyperaceen» $(11.0 \%)$, die Gramineen $(6.0 \%)$ und Artemisia (5.3\%), auf Steinschutt, sonnigem Magerrasen und in der Steppe wachsend.

Die «Cyperaceen» könnten auf ein nahes Moor deuten, aber da keine weiteren typischen MoorpflanzenPollen beobachtet werden konnten, kann man kaum auf eine bedeutende Vermoorung schließen. Diese «Cyperaceen»-Pollen können von einer unbedeutenden Vernässung nahe der Fundstelle stammen. Die reichlich vorhandenen organischen Reste und die Pollen- und Sporenarmut dieser Holzkohlenprobe erlauben mehrere Deutungsmöglichkeiten.

Betrachtet man die Holzreste und die Pollen als autochthon, so kann man auf eine Ablagerung in einen See schließen, der schwankenden Wasserstand hatte, so daß die Uferpartien hin und wieder trocken fielen und somit die Pollen teilweise (evtl. selektiv) oxydiert wurden. Da aber die Pollen und Sporen keine nennenswerten Korrosionserscheinungen zeigen, also im allgemeinen gut erhalten sind (auch die Baumpollen), kommt diese Erklärung nicht als einzige Deutungsmöglichkeit in Frage. Die vielen Holzreste und das Pollenspektrum, das auf eine Rasenvegetation deutet, passen irgendwie nicht zusammen. Demnach könnten die Holztrümmer an einem Ort mit baumarmer oder gar baumloser Vegetation zusammengeschwemmt worden sein, oder sie stellen gar mehrfach umgelagertes Material dar. Schließlich könnte man noch eine Umlagerung des gesamten organischen Materials in Erwägung ziehen. Dann ist aber die gute Erhaltung der Pollen und Sporen nicht ohne weiteres verständlich.

Obige Deutungsmöglichkeiten kann man schematisch wie folgt zusammenfassen: 


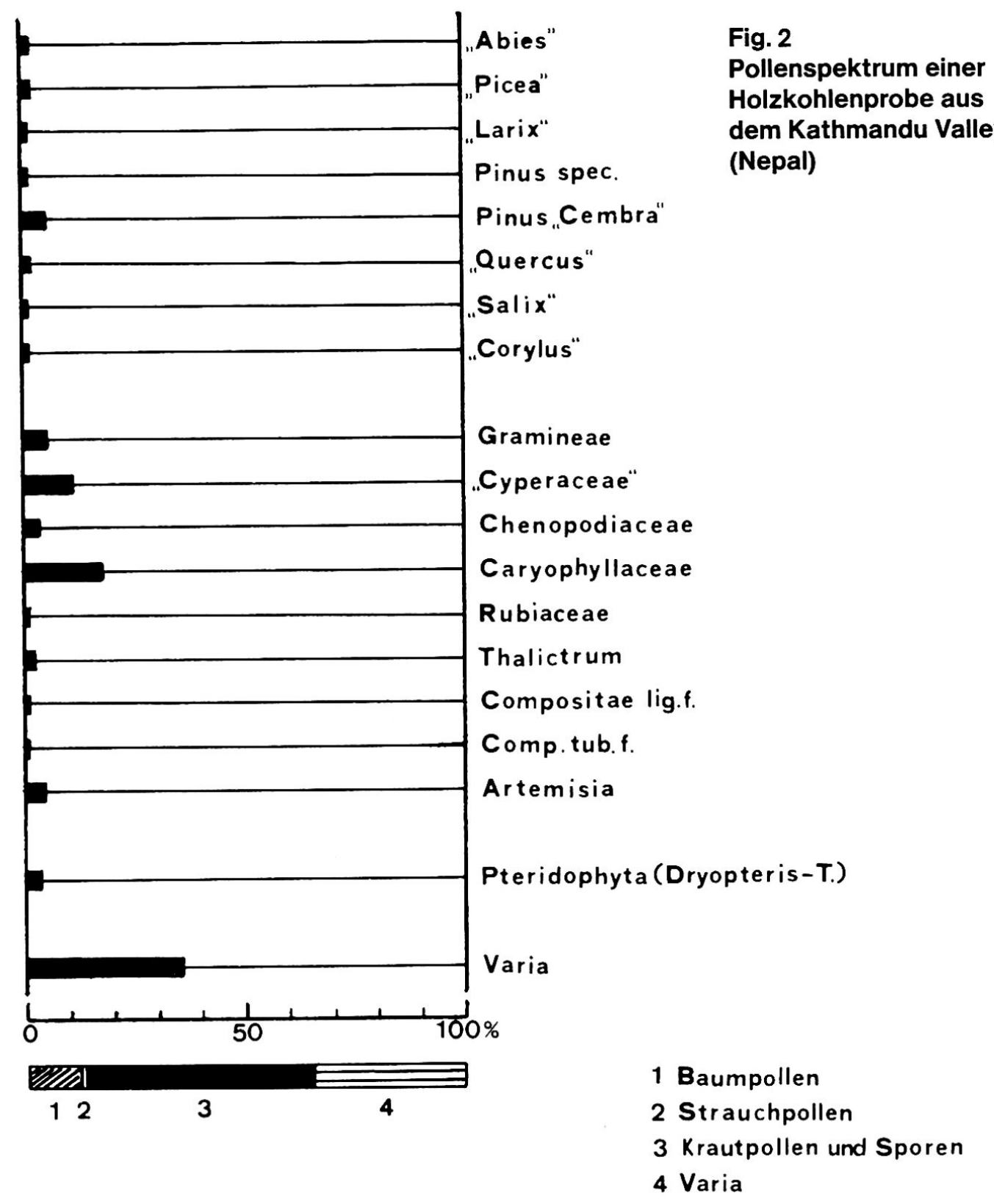

Pollensumme : 303

Pollen pro $\mathrm{cm}^{2}: 25$

Krautpollen-Typen : 10

Nadelholz-Spaltöffnungen : 4 


\begin{tabular}{|l|l|l|l|}
\hline Holzreste & Pollen und Sporen & Erklärung & Wahrscheinlichkeit \\
\hline autochthon & autochthon & $\begin{array}{l}\text { Oxydation der Pollen, besonders selektive } \\
\text { Zerstörung der Baumpollen bei Wasser- } \\
\text { standschwankungen }\end{array}$ & möglich \\
\hline autochthon & allochthon & $\begin{array}{l}\text { Oxydation der Pollen, Auslese der Pollen } \\
\text { beim Transport }\end{array}$ & gering \\
\hline allochthon & autochthon & $\begin{array}{l}\text { zusammengeschwemmtes Holzmaterial, } \\
\text { z. B. in See oder am Gleithang eines Flusses, } \\
\text { mit Rasenvegetation am Ufer }\end{array}$ & groß \\
\hline allochthon & allochthon & $\begin{array}{l}\text { verschiedene oder gleiche Herkunft der } \\
\text { Pollen und des Holzmaterials mit oder ohne } \\
\text { teilweise Zerstörung der Pollen }\end{array}$ & möglich \\
\hline
\end{tabular}

Die Beurteilung der Wahrscheinlichkeit obiger Erklärungsmöglichkeiten ist selbstverständlich mit subjektiven Momenten behaftet. Im übrigen sei betont, $\mathrm{da} ß$ es sich hier nur um eine einzelne Probe handelt, aus der man nicht zu viel Schlüsse ziehen darf.

\section{Literatur}

BEUG, H.-J. 1961: Leitfaden der Pollenbestimmung für Mitteleuropa und angrenzende Gebiete. Stuttgart.

ERdTMAn, G. 1971: Pollen Morphology and Plant Taxonomy (Angiosperms). Kopenhagen.

FAEGRI, K. und IVERSEN, J. 1964: Textbook of Pollen Analysis. 2. Aufl., Kopenhagen.

IVERSEN, J. und TROELS-SMITH, J. 1950: Pollenmorphologische Definitionen und Typen. Kopenhagen.

OBERDORFER,E. 1970: Pflanzensoziologische Exkursionsflora für Süddeutschland und die angrenzenden Gebiete. 3. Aufl., Stuttgart.

TRAUTMANN, w. 1953: Zur Unterscheidung fossiler Spaltöffnungen der mitteleuropäischen Coniferen.

Flora, Bd. 140, S. 523-533.

\section{W. A. Keller: ${ }^{14 C}$-Altersbestimmung}

Bei der untersuchten Probe handelt es sich um stark inkohltes und verfestigtes organisches Material von einheitlich grauschwarzem Aussehen. Wenige Gramm davon wurden einer chemischen Vorbehandlung unterworfen, um eventuell vorhandene kontaminierende Beimengungen zu beseitigen. Diese könnten z. B. durch die Einwirkung rezenter Huminsäuren oder rezenter bzw. fossiler Karbonatausscheidungen in das Probenmaterial gelangt sein. Selbstverständlich ist eine Verunreinigung durch makroskopisch erkennbare probenfremde Stoffe durch geeignete Entnahme der ${ }^{14} \mathrm{C}$-Probe im Labor von vornherein ausgeschlossen worden. Die anschließend mit dem gereinigten Material durchgeführten chemischen Prozesse dienten der Gewinnung von Benzol. Mit Hilfe eines Flüssigkeits-Szintillations-Zählers konnte die ${ }^{14 C}$-Restaktivität im Benzol, d. h. in dem darin enthaltenen Probenkohlenstoff bestimmt werden. Der so erhaltene Aktivitätswert ergab ein Radiokarbonalter von 19970 \pm 380 y BP, bezogen auf das Jahr 1950 und gerechnet mit der Halbwertszeit von 5570 y. Bei den Streuwerten handelt es sich um Standardabweichungen $( \pm 1 \sigma)$. Der Unterschied zwischen dem größeren positiven und dem kleineren negativen Wert ergibt sich aus dem Verlauf der Zerfalls- bzw. Altersfunktion im Bereich höherer ${ }^{14} \mathrm{C}$-Alter. 